\title{
Event Detection Time for Mobile Sensor Networks Using First Passage Processes
}

\author{
Hazer Inaltekin, Christina R. Tavoularis and Stephen B. Wicker \\ School of Electrical and Computer Engineering \\ Cornell University \\ Ithaca, NY 14853 \\ \{hi27,crt23, wicker\}@ece.cornell.edu
}

\begin{abstract}
A new technique based on first passage processes is presented as a means for calculating the detection time of an event by wireless mobile sensors located in $\mathbb{R}^{d}, d=1,2$ and 3 . Our proposed method is valid for any type of mobility model, and is amenable to a variety of extensions. We consider heterogenous networks in which sensor platforms have different mobility patterns. The second extension considers unreliable sensors with randomly distributed operational lifetimes. The second generalization is of paramount importance if the network is deployed in a hostile environment. Our main results are illustrated for random linear motion and Brownian motion. An approach for applying our theorems when sensors move according to a timehomogenous Markov process is also considered.
\end{abstract}

\section{INTRODUCTION}

In many applications, the length of time that passes until an event is detected is of critical importance. In static networks, if the amount of area covered by each sensor is equal to $A$ and the sensor locations are Poisson distributed with intensity $\lambda>$ 0 , then the fraction of area covered is equal to $1-\exp (-\lambda \cdot A)$. This is also the probability that a point target is detected by one of the sensors. In a static network, this probability remains the same over time.

In this paper, we determine event detection time probability for wireless mobile sensor networks located in $\mathbb{R}^{d}, d=1,2$ or 3. A new approach based on first passage processes is proposed to determine the time required to detect an event. This technique reduces event detection time probability calculations to that of finding the first instance in which a randomly initiated trajectory for sensor platform crosses an event boundary. Our method is valid for any kind of mobility model. We make no assumptions with regard to the type of network boundary. Our network may have no rigid, well-defined boundaries, or it may have a reflecting or an absorbing boundary. Furthermore, this method admits very natural extensions to heterogenous networks where different sensors may have different mobility patterns (e.g., linear motion and Brownian motion), and to networks with unreliable sensors having limited lifetimes. Specifically, if the network is deployed in a hostile environment such as a battlefield or the inside of a burning building, it is expected to observe unforeseen sensor failures. We illustrate the applications of our main theorems to linear motion and Brownian motion. We also outline how one can calculate event detection time probability for time-homogenous Markov processes.

\section{A. Related Work}

Coverage of wireless sensor networks and the detection of an event or an intruder are among the fundamental problems considered in the domain of wireless sensors, and have attracted considerable amount of research interest. Some of these work (e.g., [1] and [2]) deal with immobile networks, while the other (e.g., [3], [4], [5], [6] and [7]) study the coverage of mobile sensor networks.

In [1], the authors analyze the coverage of networks with immobile nodes by combining Voronoi diagrams and graph theoretical techniques. In [2], connectivity and coverage properties of an unreliable sensor grid are analyzed. Papers [3] and [4] focus on algorithmic aspects. They propose network deployment protocols to enhance the coverage of wireless mobile sensor networks after the initial placement of the sensors. Authors of [5] and [6] studied the coverage of sensor networks under random mobility patterns, which is also what we examine in this work. However, they limit themselves to very specific mobility models such as random linear mobility [5] or Brownian motion [6]. The analysis presented in these papers is based on the theory of coverage processes and only valid for infinite size networks. In contrast to them, we do not assume any specific mobility pattern (see Theorem 1). Our analysis is based on the theory of first passage processes and is valid for all finite size networks. We also show validity for infinite networks by taking the limit as the network size increases without bound. We also analyze heterogenous networks in which different sensors may have different mobility patterns (see Theorem 3), and the networks with unreliable sensors having random lifetimes (see Theorem 4). In [7], the intrusion detection problem was considered. Sensor nodes are static but a malicious agent linearly moves inside the network. They determine the first time this agent detected by the largest connected component so that message can be communicated to a sink in a multihop fashion. In this work, we do not take into account the connectivity of the network because we are looking at mobile sensor networks where each node gets connected for periods of time due to node mobility, usually within a small delay in dense networks 
(see [7]). We assume whenever any one of the sensors detects the event, it can report it to a fusion center or a sink within a bounded delay because of mobility.

\section{NeTwORK MODEL}

We consider a network of mobile sensors in $\mathbb{R}^{d}, d=1,2$ and 3. Mobile sensors are initially Poisson distributed over the network domain, which is a subset of $\mathbb{R}^{d}$, such that sensor locations $\left\{X_{i}\right\}_{i \geq 1}$ are independent. Therefore, for any Borel subset $\mathcal{A}$ of the network domain, the number of sensors in $\mathcal{A}$ obeys to a Poisson random variable with mean $\lambda|\mathcal{A}|$, where $\lambda$ is the node density, and $|\cdot|$ denotes the Lebesgue measure. If we let $S(\mathcal{A})$ to be the number of sensors in $\mathcal{A}$, then

$$
\mathbb{P}\{S(\mathcal{A})=n\}=\frac{(\lambda|\mathcal{A}|)^{n} e^{-\lambda|\mathcal{A}|}}{n !} .
$$

Let $\mathcal{B}_{R}$ represent our network domain. We consider ball shaped networks in $\mathbb{R}^{d}$, i.e., $\mathcal{B}_{R}=\left\{x \in \mathbb{R}^{d}:\|x\| \leq R\right\}$. Hence, it will be a line, disc and sphere in $\mathbb{R}^{1}, \mathbb{R}^{2}$ and $\mathbb{R}^{3}$, respectively. Let $\partial \mathcal{B}_{R}$ be the boundary of the network domain. For the general calculations, we have no assumption on the nature of the network boundary. It can be one of the following three types: (i) Hard network boundary where sensors return to the interior of the network in a manner reminiscent of that of a light wave reflecting off a mirror whenever they reach to $\partial \mathcal{B}_{R}$; (ii) Soft network boundary where sensors are stopped whenever they reach to $\partial \mathcal{B}_{R}$; (iii) No network boundary where sensors are allowed to go in and out of $\mathcal{B}_{R}$.

Each sensor is equipped with sensing capability of range $r$. Thus, a ball $\mathcal{B}(X, r)$ of radius $r$ and centered at $X$ is placed at each sensor position. Intersection of the union of such balls $\bigcup_{i} \mathcal{B}\left(X_{i}, r\right)$ with $\mathcal{B}_{R}$ represents the covered region of $\mathcal{B}_{R}$. After the initial deployment, we assume that all sensors move according to some stochastic process $\left\{M_{t}\right\}_{t>0}$, and that the motion of each sensor is independent. Consequently, the area covered by the sensors changes over time.

Under this setting, we are interested in figuring out how long it takes to detect the occurrence of an event for a given mobility model. An "event" can be a point target, an explosion, a fire or the existence of toxic chemicals and bio-hazards. For the ease of mathematical exposition, we will assume that this event affects a ball shaped region centered at the origin with radius $l$. This region will be represented by $\mathcal{B}_{l}$. We say that an event is detected once the (mobile) region, $\mathcal{B}\left(X_{i}+M_{t}, r\right)$, sensed by any one of the sensors intersects with the ball $\mathcal{B}_{l}$ (see Figure 1).

\section{Detection Time Calculations Under the General MobILITy MODEL}

In this section, we will present the calculations for finding the detection time probability of an event under the general mobility model. Let $T_{R}$ be the detection time of an event by the sensors located in $\mathcal{B}_{R}$. We first study the probability $\mathbb{P}\left\{T_{R}>t\right\}$ for $t \geq 0$. We then look at the limiting case where our network domain becomes $\mathbb{R}^{d}$. The upcoming analysis below is valid for any type of sensor mobility and previously

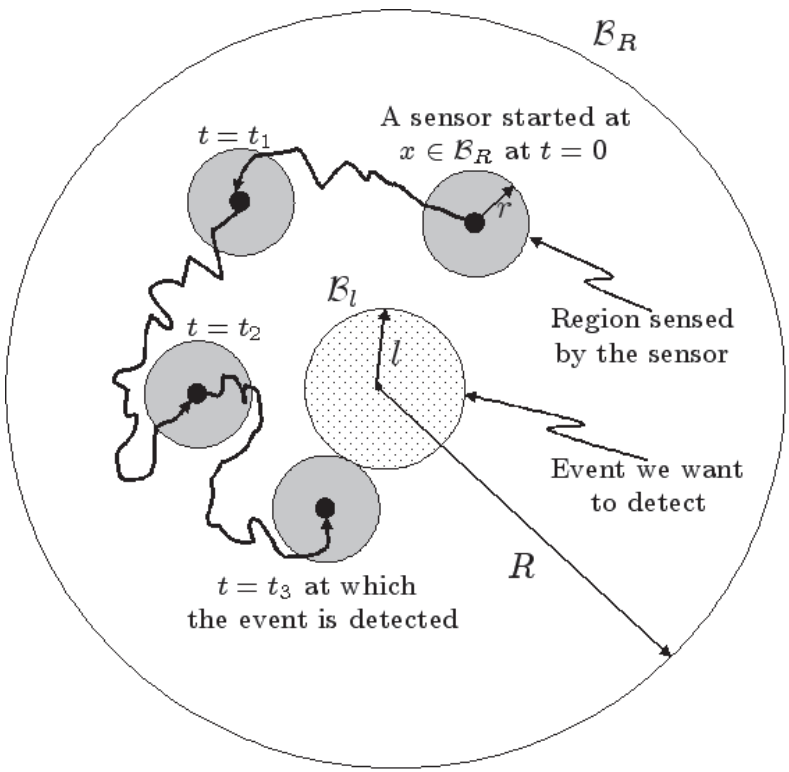

Fig. 1. A realization of the trajectory of an event detecting sensor started at $x \in \mathcal{B}_{R}$.

mentioned network boundary. Our analysis reveals that to determine $\mathbb{P}\left\{T_{R}>t\right\}$, it is enough to find the first contact time of the region sensed by a sensor node, which begins at a random position uniformly distributed over $\mathcal{B}_{R}-\mathcal{B}_{r+l}$, to the ball $\mathcal{B}_{l}$.

Theorem 1: Let $\rho=r+l$ and $p_{R, \rho}(t)$ be the probability that the sensed region of a sensor node started uniformly over the disc $\mathcal{D}(R, \rho)=\mathcal{B}_{R}-\mathcal{B}_{\rho}$ has intersected with the ball $\mathcal{B}_{l}$ by time $t$. Then,

i-) If $d=1$,

$$
\mathbb{P}\left\{T_{R}>t\right\}=\exp (-2 \lambda \rho) \cdot \exp \left(-2 \lambda(R-\rho) p_{R, \rho}(t)\right) .
$$

ii-) If $d=2$,

$\mathbb{P}\left\{T_{R}>t\right\}=\exp \left(-\lambda \pi \rho^{2}\right) \cdot \exp \left(-\lambda \pi\left(R^{2}-\rho^{2}\right) p_{R, \rho}(t)\right)$.

iii-) If $d=3$,

$\mathbb{P}\left\{T_{R}>t\right\}=\exp \left(-\frac{4}{3} \lambda \pi \rho^{3}\right) \cdot \exp \left(-\frac{4}{3} \lambda \pi\left(R^{3}-\rho^{3}\right) p_{R, \rho}(t)\right)$.

Proof: We only give the proof for $\mathbb{R}^{2}$. Proofs for the other cases $(d=1$ and $d=3)$ are similar. Let $\rho=r+l$. Consider Figure 1.

If there is a sensor inside $\mathcal{B}_{\rho}$, then $T_{R}=0$. Thus,

$$
\begin{aligned}
\mathbb{P}\left\{T_{R}>t\right\} & =\mathbb{P}\left\{S\left(\mathcal{B}_{\rho}\right)=0\right\} \mathbb{P}\left\{T_{R}>t \mid S\left(\mathcal{B}_{\rho}\right)=0\right\} \\
& =\exp \left(-\lambda \pi \rho^{2}\right) \mathbb{P}\left\{T_{R}>t \mid S\left(\mathcal{B}_{\rho}\right)=0\right\},
\end{aligned}
$$

for any $t \geq 0$. Then,

$$
\begin{gathered}
\mathbb{P}\left\{T_{R}>t\right\}=\exp \left(-\lambda \pi r^{2}\right) \sum_{n=0}^{\infty}\left(\mathbb{P}\left\{S(\mathcal{D}(R, \rho))=n \mid S\left(\mathcal{B}_{\rho}\right)=0\right\} .\right. \\
\left.\mathbb{P}\left\{T_{R}>t \mid S\left(\mathcal{B}_{\rho}\right)=0, S(\mathcal{D}(R, \rho))=n\right\}\right) \\
\stackrel{(\text { a) }}{=} \exp \left(-\lambda \pi r^{2}\right) \sum_{n=0}^{\infty} \frac{\left(\lambda \pi\left(R^{2}-\rho^{2}\right)\right)^{n} e^{-\lambda \pi\left(R^{2}-\rho^{2}\right)}}{n !}\left(1-p_{R, \rho}(t)\right)^{n}, \quad(2)
\end{gathered}
$$


where (a) follows from the spatial independence properties of the Poisson process By considering the distribution of the Poisson random variable with mean $\lambda \pi\left(R^{2}-\rho^{2}\right)\left(1-p_{R, \rho}(t)\right)$, one can simplify the summation in Equation 2 to the formula given for $d=2$.

This theorem reduces the event detection time probability calculations to finding the first hitting time of a sensor to the ball $\mathcal{B}_{\rho}$. Note that we have made no assumptions about sensor mobility and the boundary behavior of the network. As long as the sensors move independently, Theorem 1 holds.

Another important quantity of interest is the detection time $T$ of an event for infinite size networks. Intuitively, as $R$ grows to infinity, it is expected to have $\mathbb{P}\left\{T_{R}>t\right\}$ converges to $\mathbb{P}\{T>t\}$. That is, detection probability of an event is continuous at infinity. The following theorem proves this phenomenon formally. 0 .

Theorem 2: $\lim _{R \rightarrow \infty} \mathbb{P}\left\{T_{R}>t\right\}=\mathbb{P}\{T>t\}$ for any $t \geq$

Proof: We will use a coupling argument. Let $\mathcal{P}_{\lambda, R}$ be the Poisson process with intensity $\lambda$ on $\mathcal{B}_{R}$. Similarly, let $\mathcal{P}_{\lambda}$ be the Poisson process with intensity $\lambda$ on $\mathbb{R}^{d}$. We generate $\mathcal{P}_{\lambda, R}$ and $\mathcal{P}_{\lambda}$ depending on each other, i.e., they are coupled to each other. To this end, let $\mathcal{P}_{\lambda, R} \equiv \mathcal{P}_{\lambda} \cap \mathcal{B}_{R}$ for any $R>0$.

Then, first observe that the event $\left\{T_{R_{2}}>t\right\}$ is contained in the event $\left\{T_{R_{1}}>t\right\}$ whenever $R_{2} \geq R_{1}$. In other words, if the detection by any one of the sensors initially located in $\mathcal{B}_{R_{2}}$ does not occur by time $t$, this automatically implies that the detection does not occur by the sensors initially located in $\mathcal{B}_{R_{1}}$. Thus, by continuity of the probability measure from above, $\lim _{R \rightarrow \infty} \mathbb{P}\left\{T_{R}>t\right\}=\mathbb{P}\left(\bigcap_{R>0}\left\{T_{R}>t\right\}\right)$.

Let $E=\bigcap_{R>0}\left\{T_{R}>t\right\}$. It is enough to show that $E=$ $\{T>t\}$. Take any $\omega \in\{T>t\}$. Then, for any $R>0$, $T_{R}(\omega)>t$. Thus, $\omega \in E$, which implies $\{T>t\} \subseteq E$. Take now any $\omega \in E$. Assume $\omega$ does not belong to $\{T>t\}$. Then, there exists a sensor starting at some point $x \in \mathbb{R}^{d}$ such that it detects the event before time $t$. For $R \geq|x|$, this sensor lies in $\mathcal{B}_{R}$. This implies $\omega \notin\left\{T_{R}>t\right\}$ for $R \geq|x|$. Thus, $\omega \notin E$, which is a contradiction. Therefore, $\omega \in\{T>t\}$ and $E=\{T>t\}$.

One advantage of the above formalization of the event detection time problem is that Theorem 1 admits some natural and practical generalizations. We discuss two of them. In the first case, we consider the heterogenous networks in which we allow different nodes to have different mobility patterns. Formally, finite dimensional distributions of the stochastic processes $M_{t}^{(i)}$ and $M_{t}^{(j)}$ characterizing the trajectories of the mobile sensors $i$ and $j$ can differ from each other for different $i$ and $j$. For example, one of them can be Brownian motion, while the other being the simple linear mobility model. The second generalization will be to consider unreliable mobile sensors where they can die or get killed unexpectedly. In this case, we will analyze the effect of the randomness in the operational lifetime of the sensors on the event detection time probability.

\section{A. Heterogenous Networks}

To start with, let's consider the mobile sensor network in $\mathbb{R}^{2}$ containing two types of nodes, type 1 and type 2 . Type $i, i=$ 1,2 , sensors are distributed over the network domain according to the Poisson process $\mathcal{P}_{R, \lambda_{i}}$ at $t=0$, and their trajectories obey the stochastic process $M_{t}^{(i)}$ in $\mathbb{R}^{2}$ for $t>0$. Let $\lambda=$ $\lambda_{1}+\lambda_{2}$. Let $T_{R}^{(i)}$ be the detection time of the event by type $i$ sensors. Similarly, we define $p_{R, \rho}^{(i)}(t)$ as in Theorem 1 for type $i$ sensors. Since sensors move independently inside the network domain, we have $\mathbb{P}\left\{T_{R}>t\right\}=\mathbb{P}\left\{T_{R}^{(1)}>t\right\} \cdot \mathbb{P}\left\{T_{R}^{(2)}>t\right\}$. With the notations introduced in Theorem 1 , one can write $\mathbb{P}\left\{T_{R}^{(i)}>t\right\}=\exp \left(-\lambda_{i} \pi \rho^{2}\right) \exp \left(-\lambda_{i} \pi\left(R^{2}-\rho^{2}\right) p_{R, \rho}^{(i)}(t)\right)$. As a results, $\mathbb{P}\left\{T_{R}>t\right\}$ becomes equal to:

$$
\begin{aligned}
& \mathbb{P}\left\{T_{R}>t\right\}= \\
& \quad \exp \left(-\lambda \pi \rho^{2}\right) \cdot \exp \left(-\pi\left(R^{2}-\rho^{2}\right) \sum_{i=1}^{2} \lambda_{i} \cdot p_{R, \rho}^{(i)}(t)\right) .
\end{aligned}
$$

Through similar calculations, one can extend the Equation 3 to the networks in $\mathbb{R}^{d}, d=1,2,3$, with arbitrary number of sensor types as follows.

Theorem 3: Consider a mobile sensor network in $\mathbb{R}^{d}, d=$ $1,2,3$, with sensor types $i=1,2, \ldots, n$. Each sensor type is distributed over the network domain according to a Poisson process with intensity $\lambda_{i}$. Let $\lambda=\sum_{i=1}^{n} \lambda_{i}$. Then, it is enough to replace the first hitting time probability $p_{R, \rho}(t)$ in Theorem 1 with $\frac{1}{\lambda} \sum_{i=1}^{n} \lambda_{i} p_{R, \rho}^{(i)}$ in order to determine $\mathbb{P}\left\{T_{R}>t\right\}$.

\section{B. Sensors with Limited Lifetime}

There are several practical reasons to study the event detection times for networks having sensors with limited (random) lifetime. Sensor nodes, especially the mobile ones, have limited operational battery lifetimes. The process of sensing, transmitting and receiving packets as well as moving inside the network domain drain sensors' battery energy. The second reason is the consideration of harsh environment factors. Sensor networks may be deployed in very hostile environments such as in battlefields, or inside a building in case of a fire Under these conditions, unexpected sensor failures may occur. The third reason is the possibility of the existence of agents or enemies trying to jam the sensing capability of our network. A sensor that is successfully jammed by an enemy can be disabled.

These reasons motivate us to consider networks with sensors having limited lifetime. To this end, let $\zeta$ be the random variable representing the sensor lifetime. Mobile sensors are initially distributed over the network domain according to $\mathcal{P}_{R, \lambda}$, and then move according to $\left\{M_{t}\right\}_{t>0}$ until $\zeta$. Stochastic process $\widetilde{M}_{t}$ characterizing the sensor mobility is equal to $M_{t}$ if $t<\zeta$, and to $\partial$ if $t \geq \zeta$ for some $\partial \notin \mathbb{R}^{d}$ "coffin" state. Let $\tilde{\tau}$ and $\tau$ be the random variables representing the first time a sensor with sensing range $r$ detects the event $\mathcal{B}_{l}$ when it moves according to $\widetilde{M}_{t}$ and $M_{t}$ respectively, and its initial position is chosen randomly uniformly over the disc 
$\mathcal{D}_{R, \rho}=\mathcal{B}_{R}-\mathcal{B}_{\rho}$, where $\rho=r+l$. Let $\tilde{p}_{R, \rho}(t)=\mathbb{P}\{\tilde{\tau} \leq t\}$ and $p_{R, \rho}(t)=\mathbb{P}\{\tau \leq t\}$. Then, we have:

$$
\begin{aligned}
\tilde{p}_{R, \rho}(t) & =\mathbb{P}\{\tilde{\tau} \leq t, \zeta>t\}+\mathbb{P}\{\tilde{\tau} \leq t, \zeta \leq t\} \\
& =\mathbb{P}\{\zeta>t\} \mathbb{P}\{\tilde{\tau} \leq t \mid \zeta>t\}+\mathbb{P}\{\tilde{\tau} \leq t, \zeta \leq t\} \\
& \stackrel{(\mathrm{a})}{=} \mathbb{P}\{\zeta>t\} p_{R, \rho}(t)+\mathbb{P}\{\tilde{\tau} \leq t, \zeta \leq t\}
\end{aligned}
$$

where (a) follows from the fact that the trajectory of a sensor is equal to $M_{t}$ in distribution given that it has not died yet. Let $f_{\zeta}(t)$ be the probability density function of $\zeta .{ }^{1}$ Then, we can compute the second probability as follows:

$$
\begin{aligned}
\mathbb{P}\{\tilde{\tau} \leq t, \zeta \leq t\} & =\int_{0}^{t} f_{\zeta}(s) \mathbb{P}\{\tilde{\tau} \leq t \mid \zeta=s\} d s \\
& =\int_{0}^{t} f_{\zeta}(s) \mathbb{P}\{\tilde{\tau} \leq s \mid \zeta=s\} d s \\
& =\int_{0}^{t} f_{\zeta}(s) p_{R, \rho}(s) d s \\
& =\mathbb{E}\left[p_{R, \rho}(\zeta) \cdot \mathbb{1}_{\{\zeta \leq t\}}\right] .
\end{aligned}
$$

If we combine Equations 4 and 5 , we obtain $\tilde{p}_{R, \rho}(t)=$ $\mathbb{E}\left[p_{R, \rho}(t \wedge \zeta)\right]$, where $t \wedge \zeta$ represents the minimum of $t$ and $\zeta$. If we replace $p_{R, \rho}(t)$ with $\tilde{p}_{R, \rho}(t)$ in Theorem 1 , we obtain the event detection time probability for the mobile sensor networks with unreliable sensors having limited lifetimes as stated in the following theorem.

Theorem 4: Consider a mobile sensor network in $\mathbb{R}^{d}, d=$ $1,2,3$, with unreliable sensor nodes having limited lifetimes. Let sensor lifetimes be distributed according to the random variable $\zeta$. Sensors are initially Poisson distributed over the network domain with intensity $\lambda$, and then move according to $\left\{M_{t}\right\}_{0 \leq t \leq \zeta}$ until $\zeta$. For such networks, it is enough to replace $p_{R, \rho}(t)$ with $\mathbb{E}\left[p_{R, \rho}(t \wedge \zeta)\right]$ in Theorem 1 to determine $\mathbb{P}\left\{T_{R}>\right.$ $t\}$.

Several other extensions of Theorem 1 combining heterogenous networks with unreliable mobile sensor nodes are also possible. They are left to the reader due to space limitations.

\section{One Dimensional Networks}

For the rest of the paper, we illustrate the applications of the main theorems obtained in section III on more concrete mobility models such as random linear mobility model and Brownian motion. We start our analysis with 1-D sensor networks containing sensors which move according to Brownian motion. The main steps of the analysis for this case can be extended to any Markov process on the line. We will also briefly mention this extension.

\section{A. Brownian Motion on the Line}

Consider a 1-D mobile sensor network in which sensors move independently according to a Brownian motion $\left\{B_{t}\right\}_{t \geq 0}$ with zero drift and variance parameter $\sigma^{2}>0$. An event occurs at the origin and affects the area $(-l, l)$. Sensing range of sensors is $r$. If a sensor is located at $x$, it senses the region

\footnotetext{
${ }^{1}$ If $\zeta$ does not have a density, then one can use its distribution function in rest of the analysis. The same result continues to hold.
}

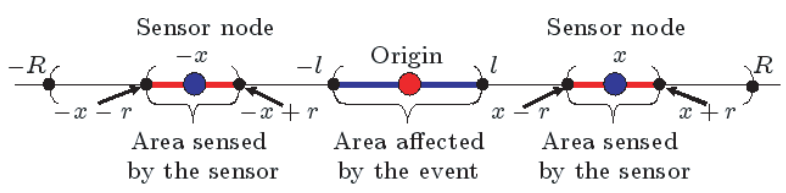

Fig. 2. Detection of the event for 1-dim networks.

$(x-r, x+r)$ (See Figure 2). Therefore, whenever any one of the sensors becomes closer than $\rho=l+r$ to the origin, the event is detected. We will now briefly present the calculations based on the Laplace transform techniques for finding the first detection time of the event by a sensor starting its motion at $x \geq \rho$ at time 0 . The main steps of this analysis will also be valid for any continuous Markov process on the line. Our calculations will be based on the analysis given in [8].

Let $q(x, s ; y, t)$ be the transition probability density function (TPDF) of $B_{t}$, where $t \geq s \geq 0$. Intuitively, it is the probability of $B_{t}$ being in $(y-d y, y+d y)$ given $B_{s}=x$, i.e., $q(x, s ; y, t)=\mathbb{P}\left\{B_{t} \in(y-d y, y+d y) \mid B_{s}=x\right\} .{ }^{2}$ For the rest of the analysis, we will consider a sensor located at an arbitrary position $\|x\| \geq \rho$ at time $t=0$. Let $\tau$ be the first time it reaches $\rho$, and $f_{\tau}^{x}(t)$ be its probability density function (PDF). Pick a point $y \in(0, \rho)$. To reach $y$ at time $t$, the sensor first arrives $\rho$ at time $\tau$, and in the remaining time $t-\tau$, it makes its way to $y$. As a result, one can obtain by using strong Markov property that $q(x, 0 ; y, t)=\int_{0}^{t} f_{\tau}^{x}(s) \cdot q(\rho, 0 ; y, t-s) d s$. When viewed as a function of $t$, the integral is the convolution of $f_{\tau}^{x}(t)$ and $q(\rho, 0 ; y, t)$. Hence, the Laplace transform $\tilde{f}_{\tau}^{x}(w)$ of $f_{\tau}^{x}(t)$ is obtained as

$$
\tilde{f}_{\tau}^{x}(w)=\frac{\tilde{q}(x, 0 ; y, w)}{\tilde{q}(\rho, 0 ; y, w)},
$$

where $\tilde{q}$ is the Laplace transform of $q . q(x, s ; y, t)$ for Brownian motion is equal to $\frac{1}{\sqrt{2 \pi t \sigma^{2}}} \exp \left(-\frac{|x-y|^{2}}{2|t-s| \sigma^{2}}\right)$. After taking the Laplace transform of $q(x, 0 ; y, t)$ with respect to $t$, one obtains

$$
\tilde{q}(x, 0 ; y, w)=\frac{1}{\sqrt{2 \sigma^{2} w}} \exp \left(-\frac{\sqrt{2 w}|x-y|}{\sigma}\right) .
$$

By using Equations 6, 7 and the symmetry properties of the Brownian motion, one obtains $\tilde{f}_{\tau}^{x}(w)=\exp \left(-\frac{\sqrt{2 w}|x-\rho|}{\sigma}\right)$. This is the characteristic function corresponding to the stable distribution with parameter $\frac{1}{2}$. After inversion, we have $f_{\tau}^{x}(t)=\frac{|x-\rho|}{\sqrt{2 \pi t^{3} \sigma^{2}}} \cdot \exp \left(-\frac{|x-\rho|^{2}}{2 \sigma^{2} t}\right)$. Integrating this PDF, we can obtain the detection probability for a sensor started at $x$.

$$
\mathbb{P}^{x}\{\tau \leq t\}=\int_{0}^{t} f_{\tau}^{x}(s) d s=\operatorname{Erfc}\left(\frac{|x-\rho|}{\sqrt{2 t \sigma^{2}}}\right) .
$$

In order to calculate $p_{R, \rho}(t)$, one needs to replace $x$ in the above equation with a uniformly distributed random variable $X$ over $(-R,-\rho) \cup(R, \rho)$, and take one more expectation over $X$.

$p_{R, \rho}(t)=\mathbb{E}\left[\mathbb{P}^{X}\{\tau \leq t\}\right]=\frac{1}{R-\rho} \int_{\rho}^{R} \operatorname{Erfc}\left(\frac{x-\rho}{\sqrt{2 t} \sigma}\right) d x$.

\footnotetext{
${ }^{2}$ Formally, $q(x, s ; y, t)=\frac{\mathrm{d}}{\mathrm{d} y} \mathbb{P}\left\{B_{t} \leq y \mid B_{s}=x\right\}$.
} 
Putting the expression of $p_{R, \rho}(t)$ into the formula given for 1-D networks in Theorem 1, we obtain

$\mathbb{P}\left\{T_{R}>t\right\}=\exp (-2 \lambda \rho) \exp \left(-2 \lambda \int_{\rho}^{R} \operatorname{Erfc}\left(\frac{x-\rho}{\sqrt{2 t} \sigma}\right)\right) d x$

By taking $R$ to infinity and using Theorem 2, we also obtain the detection time probability for infinite size networks:

$$
\mathbb{P}\{T>t\}=\exp (-2 \lambda \rho) \exp \left(-\frac{2 \lambda \sigma \sqrt{2 t}}{\sqrt{\pi}}\right) .
$$

\section{B. Large $t$ Behavior of the Detection Probability}

For other mobility models different than Brownian motion, it may not be possible to obtain the inverse Laplace transform for $\tilde{f}_{\tau}^{x}(w)$. In these cases, one can invert it numerically to obtain $p_{R, \rho}(t)$. However, if we are interested in the long time behavior of the probability $\mathbb{P}\left\{T_{R}>t\right\}$ as $t \rightarrow \infty$, the more eloquent way is to use Tauberian theorems to determine the asymptotic behavior for $\mathbb{P}\left\{T_{R}>t\right\}$. Very briefly, Tauberian theorems say that behavior of the Laplace transform near the origin uniquely determines the behavior of the function at infinity. We will illustrate the application of Tauberian theorems for the Brownian motion case. To this end, we have:

$$
\begin{aligned}
\mathcal{L}\{2 \lambda(R-\rho) & \left.p_{R, \rho}(t)\right\} \\
& =2 \lambda(R-\rho) \mathcal{L}\left\{\mathbb{E}\left[\int_{0}^{t} f_{\tau}^{X}(s) d s\right]\right\} \\
& \stackrel{(\mathrm{a})}{=} 2 \lambda(R-\rho) \mathbb{E}\left[\mathcal{L}\left\{\int_{0}^{t} f_{\tau}^{X}(s) d s\right\}\right] \\
& \stackrel{(\mathrm{b})}{=} 2 \lambda(R-\rho) \mathbb{E}\left[\frac{\tilde{f}_{\tau}^{X}(w)}{w}\right] \\
& =\frac{2 \lambda}{w} \int_{\rho}^{R} \exp \left(-\frac{\sqrt{2 w}(x-\rho)}{\sigma}\right) d x \\
& =\frac{2 \lambda \sigma}{\sqrt{2} w^{\frac{3}{2}}} \cdot\left(1-\exp \left(-\frac{\sqrt{2 w}(R-\rho)}{\sigma}\right)\right),
\end{aligned}
$$

where (a) follows from Fubini's theorem, and (b) is the integration formula for Laplace transform. Let's consider infinite size networks, and let $p_{\rho}(t)=\lim _{R \rightarrow \infty} 2 \lambda(R-\rho) p_{R, \rho}(t)$. Note that $p_{\rho}(t)$ is not probability, and may be bigger than 1 . Laplace transform $\tilde{p}_{\rho}(w)$ of $p_{\rho}(t)$ is equal to $\frac{2 \lambda \sigma w^{-\frac{3}{2}}}{\sqrt{2}}$. $^{3}$ Behavior of $\tilde{p}_{\rho}(w)$ around zero determines the behavior of the detection time probability at infinity. Since $\tilde{p}_{\rho}(w)$ is asymptotic to $\frac{2 \lambda \sigma w^{-\frac{3}{2}}}{\sqrt{2}}$ as $w \rightarrow 0$, we have $p_{\rho}(t)$ asymptotic to $\frac{2 \lambda \sigma \sqrt{2 t}}{\sqrt{\pi}}$ as $t \rightarrow \infty$ (See chapter 13 of [8], Theorem 4). This is exactly the same exponent appearing in Equation 10.

\section{Reflecting and Absorbing Network Boundaries}

Following exactly the same analysis, one can obtain expressions of the detection time probability for networks with different boundary behavior. For example, consider a 1-D network with reflecting boundaries $\{-R, R\}$. Let's consider a sensor node initially located at $x \in(\rho, R)$ and moving

\footnotetext{
${ }^{3}$ The change of limit and integration can be justified by using dominated convergence theorem.
}

according to $B_{t}$. The first time it detects the event is equal to the first time a Brownian motion particle reflected at $R$ becomes closer than $\rho$ to the origin. TPDF for the Brownian motion started at $x \in(\rho, R)$ and reflected at $R$ is equal to $q_{+}(x, 0 ; y, t)=q(x, 0 ; y, t)+q(x, 0 ; 2 R-y, t)$. As a result, if the above analysis is repeated with $q_{+}(x, 0 ; y, t)$ in stead of $q(x, 0 ; y, t)$, we obtain the detection time probability for networks with reflecting boundaries. If the boundary $\{-R, R\}$ is absorbing, then it is enough to use $q_{-}(x, 0 ; y, t)=$ $q(x, 0 ; y, t)-q(x, 0 ; 2 R-y, t)$ for TPDF, and repeat the same analysis given above.

\section{Calculations for General Time-Homogenous Markov Pro- cesses}

We will now briefly present how one can proceed to calculate the detection time probability for a network having sensors moving according to a Markov process $\left\{M_{t}\right\}_{t>0}$. TPDF $q(x, s ; y, t)$ of a Markov process satisfies the Kolmogorov's backward equation (KBE) $\frac{\partial q}{\partial t}=\frac{1}{2} \sigma^{2}(x) \frac{\partial^{2} q}{\partial x^{2}}+\mu(x) \frac{\partial q}{\partial x}$ with appropriate boundary conditions (see [9]). $\sigma^{2}(x)$ and $\mu(x)$ are called infinitesimal parameters of the process, and defined as $\mu(x)=\lim _{h \downarrow 0} \mathbb{E}\left[M_{t+h}-M_{t} \mid M_{t}=x\right]$ and $\sigma^{2}(x)=$ $\lim _{h \downarrow 0} \mathbb{E}\left[\left(M_{t+h}-M_{t}\right)^{2} \mid M_{t}=x\right]$. They only depend on $x$ due to time-homogeneity. For example, $\sigma^{2}(x)=\sigma^{2}$ and $\mu=0$ for Brownian motion without drift. Solving KBE, we obtain TPDF $q(x, s ; y, t)$ of the process, and then follow the same steps as in Brownian motion case. Large time behavior of the detection probability can also be obtained by using Tauberian theorems.

\section{Linear Motion In $\mathbb{R}^{2}$}

We will illustrate another application of our main Theorem 1. Consider linearly moving sensors in $\mathbb{R}^{2}$. Under this mobility model, a sensor chooses a random direction $\Theta \in[0,2 \pi]$ and moves with a constant speed $v$ in this direction. A sensor senses the event whenever it becomes closer than $\rho$ to the origin. Let $\tau$ be the first time a sensor started randomly uniformly over the disc $\mathcal{D}_{R, \rho}=\mathcal{B}_{R}-\mathcal{B}_{\rho}$ senses the event. Then, $p_{R, \rho}(t)=\mathbb{P}\{\tau \leq t\}$ can be calculated by first conditioning on the initial position $M_{0}$ of the sensor, and then taking one more expectation over all $M_{0}$ :

$$
p_{R, \rho}(t)=\mathbb{E}\left[\mathbb{P}\left\{\tau \leq t \mid M_{0}\right\}\right] .
$$

Consider the Figure 3. If the sensor chooses the wrong direction, i.e., $\Theta \notin\left[-\alpha\left(M_{0}\right), \alpha\left(M_{0}\right)\right]$, then it cannot detect the event. On the other hand, if it chooses the correct direction, i.e., $\Theta \in\left[-\alpha\left(M_{0}\right), \alpha\left(M_{0}\right)\right]$, it will detect the event eventually but it takes sometime to reach $\mathcal{B}_{\rho}$. Considering all the cases and using $f_{\left\|M_{0}\right\|}(x)=\frac{2 x}{R^{2}-\rho^{2}} \mathbb{1}_{\{\rho \leq x \leq R\}}$ for the PDF of $\left\|M_{0}\right\|$, the expression for $p_{R, \rho}(t)$ can be obtained as in Equation 12. We will not provide the details of the calculations due to space limitations.

$$
\begin{aligned}
p_{R, \rho}(t) & =\frac{1}{\pi\left(R^{2}-\rho^{2}\right)}\left[\rho \sqrt{R^{2}-\rho^{2}}+R^{2} \arcsin \left(\frac{\rho}{R}\right)-\frac{\pi \rho^{2}}{2}\right] \\
& -\frac{\mathbb{1}\left\{t v \leq \sqrt{\left.R^{2}-\rho^{2}\right\}}\right.}{\pi\left(R^{2}-\rho^{2}\right)}\left[\rho \sqrt{R^{2}-\rho^{2}}+R^{2} \arcsin \left(\frac{\rho}{R}\right)-2 \rho t v-\frac{\pi \rho^{2}}{2}\right](12)
\end{aligned}
$$




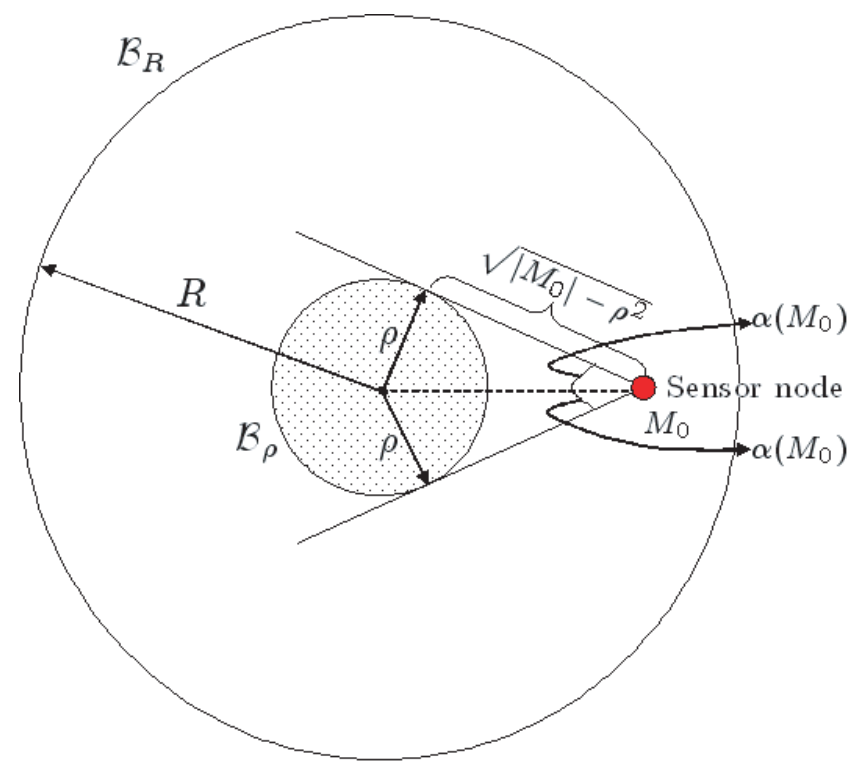

Fig. 3. A sensor initially located at $M_{0}$ and moving according to linear motion.

Note that as $t \rightarrow \infty, p_{R, \rho}(t) \rightarrow \mathbb{P}\{\tau<\infty\}=$ $\frac{1}{\pi} \frac{1}{R^{2}-\rho^{2}}\left[\rho \sqrt{R^{2}-\rho^{2}}+R^{2} \arcsin \left(\frac{\rho}{R}\right)-\frac{\pi \rho^{2}}{2}\right]$. Hence, a sensor may never detect the event with some positive probability. This further implies that for a finite size network, event detection time can be infinite with positive probability for linear mobility. This happens when all of the sensors choose a wrong direction and miss the event. $\mathbb{P}\left\{T_{R}>t\right\}$ for linearly moving sensors can be obtained by plugging Equation 12 to the formula for 2-D networks in Theorem 1. In light of Theorem 2 , detection probability $\mathbb{P}\{T>t\}$ for infinite size networks can be obtained by taking the limit $\mathbb{P}\left\{T_{R}>t\right\}$ as $R$ goes to infinity.

$\mathbb{P}\{T>t\}=\lim _{R \rightarrow \infty} \mathbb{P}\left\{T_{R}>t\right\}=\exp \left(-\lambda \pi \rho^{2}\right) \exp (-2 \lambda \rho t v)$.

Note that $T$ is exponentially distributed on the event that there is no sensor located in $\mathcal{B}_{\rho}$ at time 0 . A similar result was also obtained in [5] by using coverage processes. Several other extensions of this analysis to heterogenous networks with sensors having different mobility models and sensors with limited lifetimes are also possible by using Theorems 3 and 4.

\section{Time-homogenous Markov Processes on $\mathbb{R}^{d}$}

We will now briefly mention how one can proceed to calculate the detection time probability for time-homogenous Markov processes $M_{t}=\left(M_{t}^{(1)}, \ldots, M_{t}^{(d)}\right)$ on $\mathbb{R}^{d}$. We will construct $M_{t}$ by taking independent copies of a 1-D timehomogenous Markov process $M_{t}^{(i)}$ and declaring it to be the $i^{\text {th }}$ component of $M_{t}$. TPDF $q_{i}\left(x_{i}, s ; y_{i}, t\right)$ of each component of $M_{t}$ can be obtained by solving $\mathrm{KBE}$. TPDF of $M_{t}$ is then just the multiplication of individual $q_{i}$ 's: $q(\mathbf{x}, s ; \mathbf{y}, t)=$ $\prod_{i=1}^{d} q_{i}\left(x_{i}, s ; y_{i}, t\right)$. Now, consider a point $\mathbf{y}=\left(y_{1}, \ldots, y_{d}\right)$ with $\|\mathbf{y}\|<\rho$. In order to reach a small ball $\mathcal{B}(\mathbf{y}, \epsilon)$ around $\mathbf{y}$, a sensor first needs to reach to the boundary $\partial \mathcal{B}_{\rho}$ of the ball $\mathcal{B}_{\rho}$ at time $\tau$, and then makes its way to $\mathcal{B}(\mathbf{y}, \epsilon)$ in the remaining time $t-\tau$. As a result, Laplace transform of $f_{\tau}^{x}(t)$ can be obtained through an analysis similar to the one given in section IV. One exception is that the point $M_{t}$ hits on the surface of the sphere $\mathcal{B}_{\rho}$ is random in this case. Therefore, we need to appropriately scale the denominator of Equation 6 with the harmonic measure $\mu(\theta)$ induced by $M_{\tau}$ on $\partial \mathcal{B}_{\rho}$. Finally, the expression for $\tilde{f}_{\tau}^{x}(w)$ can be given as:

$$
\tilde{f}_{\tau}^{x}(w)=\frac{\tilde{p}(\mathbf{x}, 0 ; \mathbf{y}, w)}{\int_{\partial \mathcal{B}_{\rho}} \tilde{p}(\theta, 0 ; \mathbf{y}, w) d \mu(\theta)} .
$$

After obtaining $\tilde{f}_{\tau}^{x}(w)$, one can proceed as in Brownian motion case to obtain $p_{R, \rho}(t)$ and $\mathbb{P}\left\{T_{R}>t\right\}$.

\section{CONCLUSION}

In this paper, we studied the event detection probability for wireless mobile sensor networks. In particular, we analyzed the probability $\mathbb{P}\left\{T_{R}>t\right\}$ of an event going undetected until time $t$. We proposed a general method for calculating $\mathbb{P}\left\{T_{R}>t\right\}$ based on first passage processes. We showed that under any mobility model, calculating $\mathbb{P}\left\{T_{R}>t\right\}$ reduces to finding the first time of a sensor hits to the region affected by the event - Theorem 1. Our general method also admits some natural extensions such as heterogenous networks in which different sensors may have different mobility patterns and networks having sensors with limited lifetimes - Theorems 3 and 4. We then illustrated applications of our main theorems on concrete mobility patterns such as Brownian motion and random linear mobility. We also outlined how one can proceed to calculate event detection time under more general mobility models such as time-homogenous Markov processes on $\mathbb{R}^{d}, d=1,2,3$. A variety of mobile sensor network scenarios, in addition to the ones presented, may be analyzed using the tools and techniques presented in this paper.

\section{REFERENCES}

[1] Meguerdichian S., Koushanfar F., Potkonjak M. and Srivastava M. B., Coverage Problems in Wireless Ad-hoc Sensor Networks, IEEE Infocom, 2001

[2] Shakkottai S., Srikant R. and Shroff N., Unreliable Sensor Grids: Coverage, Connectivity and Diameter, IEEE Infocom, 2003.

[3] Zou. Y and Chakrabarty K., Sensor Deployment and Target Localization Based on Virtual Forces, IEEE Infocom 2003.

[4] Wang G., Cao G. and Porta L. T., Movement-assisted Sensor Deployment, IEEE Infocom 2004.

[5] Liu B., Brass P., Dousse O., Nain P. and Towsley D., Mobility Improves Coverage of Sensor Networks, Mobihoc 2005.

[6] Kesidis G., Konstantopoulos T., Phoha S., Surveillance Coverage of Sensor Networks under a Random Mobility, Proc. IEEE Sensors, 2003.

[7] Dousse 0., Tavoularis C. and Thiran P., Delay of Intrusion Detection in Wireless Sensor Networks, Mobihoc 2006.

[8] William Feller, An Introduction to Probability Theory and Its Applications, Willey Series in Probability and Mathematical Statistics, Vol.II, 1970.

[9] Karlin S. and Taylor M. H., A Second Course in Stochastic Processes, Academic Press, 1981. 